\title{
Electric Field Standing Wave Effects in FT-IR Transflection Spectra of biological tissue sections: simulated models of experimental variability
}

Tomasz P. Wrobel

Jagiellonian University Cracow

Barbara Wajnchold

Jagiellonian University Cracow

Hugh J. Byrne

Technological University Dublin, hugh.byrne@tudublin.ie

See next page for additional authors

Follow this and additional works at: https://arrow.tudublin.ie/biophonart

Part of the Analytical, Diagnostic and Therapeutic Techniques and Equipment Commons, Biochemistry, Biophysics, and Structural Biology Commons, Biotechnology Commons, and the Physics Commons

\section{Recommended Citation}

Wrobel, T. P. et al (2013) Electric Field Standing Wave Effects in FT-IR Transflection Spectra of biological tissue sections: simulated models of experimental variability", Vibrational Spectroscopy, 69, pp.84-92. http://dx.doi.org/10.1016/j.vibspec.2013.09.008

This Article is brought to you for free and open access by the DIT Biophotonics and Imaging at ARROW@TU Dublin. It has been accepted for inclusion in Articles by an authorized administrator of ARROW@TU Dublin. For more information, please contact arrow.admin@tudublin.ie, aisling.coyne@tudublin.ie,gerard.connolly@tudublin.ie. Funder: HEA

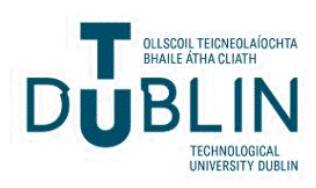


Authors

Tomasz P. Wrobel, Barbara Wajnchold, Hugh J. Byrne, and Malgorzata Baranska

This article is available at ARROW@TU Dublin: https://arrow.tudublin.ie/biophonart/10 


\title{
Electric Field Standing Wave Effects in FT-IR Transflection Spectra of biological tissue sections: simulated models of experimental variability
}

\author{
Tomasz P. Wrobel ${ }^{\mathrm{a}, \mathrm{b}}$, Barbara Wajnchold ${ }^{\mathrm{c}}$, Hugh J. Byrne ${ }^{\mathrm{d} *}$, Malgorzata Baranska ${ }^{\mathrm{a}, \mathrm{b} *}$ \\ ${ }^{a}$ Faculty of Chemistry, Jagiellonian University, 3 Ingardena Str., 30-060 Krakow, Poland, e-mail: \\ baranska@chemia.uj.edu.pl \\ bJagiellonian Centre for Experimental Therapeutics (JCET), Jagiellonian University, 14 Bobrzynskiego \\ Str., 30-348, Krakow, Poland \\ ${ }^{c}$ Marian Smoluchowski Institute of Physics, Jagiellonian University, Reymonta 4 Str., Krakow, Poland \\ ${ }^{\mathrm{d}}$ Focas Research Institute, Dublin Institute of Technology (DIT), Camden Row, Dublin 8, Ireland, e- \\ mail: hugh.byrne@dit.ie
} 10

\begin{abstract}
The so-called electric field standing wave effect (EFSW) has recently been demonstrated to significantly distort FT-IR spectra acquired in a transflection mode, both experimentally and in ${ }_{20}$ simulated models, bringing into question the appropriateness of the technique for sample characterization, particularly in the field of spectroscopy of biological materials. The predicted effects are most notable in the regime where the sample thickness is comparable to the source wavelength. In this work, the model is extended to sample thicknesses more representative of biological tissue sections and to include typical experimental factors which are demonstrated to reduce the predicted ${ }_{25}$ effects. These include integration over the range of incidence angles, varying degrees of coherence of the source and inhomogeneities in sample thickness. The latter was found to have the strongest effect on the spectral distortions and, with inhomogeneities as low as $10 \%$ of the sample thickness, the predicted distortions due to the standing wave effect are almost completely averaged out. As the majority of samples for biospectroscopy are prepared by cutting a cross section of tissue resulting in a ${ }_{30}$ high degree of thickness variation, this finding suggests that the standing wave effect should be a minor distortion in FT-IR spectroscopy of tissues. The study has important implications not only in optimization of protocols for future studies, but notably for the validity of the extensive studies which have been performed to date on tissue samples in the transflection geometry.
\end{abstract}

Keywords: Fourier Transform infrared spectroscopy, tissues, transflection, electric field standing wave effect, simulation, sample inhomogeneity, biospectroscopy

${ }_{40}$ Correspondence to:

e-mail: baranska@chemia.uj.edu.pl tel.+48 126632253 , fax.+48 126340515 


\section{Introduction}

There has been considerable debate over the past decade (and more) with regard to the validity of FT${ }_{45}$ IR spectroscopic measurements, as physical/optical effects due to chemical and physical inhomogeneities have been shown to introduce spectral artefacts. The issues have been highlighted in the field of biospectroscopy, but are pertinent to the use of IR spectroscopy in a broad range of applications, particularly for samples with significant chemical and physical inhomogeneity. Resonant and nonresonant reflection and scattering have been identified as the source of some of these effects, ${ }_{50}$ and correction algorithms have been developed to account for them [1-7].

FT-IR imaging has already proven its worth in many biological applications including measurement for both cells and tissues[8-16]. However, measurement in transmission mode requires the use of relatively expensive IR transparent substrates (e.g. $\mathrm{CaF}_{2}$ ), leading to the popularity of the transflection mode of measurement performed on MirrIR Kevley Glass, which has already been successfully ${ }_{55}$ applied in numerous biomedical imaging studies [2, 4, 17-20]. Since protocols for correction of spectral artefacts have been developed, the extensive use of transflection measurements appeared acceptable. However, recent advances in understanding the complete optical system, including the sources of such artefacts, has led to identification of an electric field standing wave effect (EFSW) by Brooke et al. [21], substantiated by further important investigations by Filik et al. [22, 23] and Bassan ${ }_{60}$ et al. [24]

The latter work simulated a multilayer system and modeled spectra of a hypothetical absorber with sample thickness varying between 0.5 and $4.5 \mu \mathrm{m}$. Subsequently, the hypothetical absorber was replaced by experimental cytosine spectra in the model and compared with experimental data in the thickness range of $0.5-1.5 \mu \mathrm{m}$. The simulation of the EFSW gave a very valuable insight in the ${ }_{65}$ dependences of the phenomenon on physical variables such as the sample thickness, and furthermore demonstrated the significant implications this can have on, for example biological tissue classification for disease diagnostics. Notably, however, the predicted effects were significantly higher than those observed experimentally for cytosine films spectra (comparing Figures 5 and Figure 11 of reference [24]). This suggests therefore that the model did not take into account all experimental parameters of

${ }_{70}$ significance for the final spectral outcome, and that the implications of the ESFM for the use of FTIR in the transflection mode may not be as dramatic as initially indicated by the simulation.

The real system of the transflection experiment is very complex and nontrivial to simulate. For this work, it was decided to build a simple model which could help to understand the impact of the EFSW in experimental data, and also to compare with the simulations of Filik et al.[22] and Bassan et al.[24]. ${ }_{75}$ Initially, the predictions are compared with those of the literature, after which, the model is broadened to include more realistic experimental parameters. Those identified as having a possible averaging effect of the EFSW are:

- the range of incidence angles (in 15x Cassegrain objectives typically 35-45 degrees)

- the degree of coherence of the incident light (all simulations were previously done with only a single phase source)

- inhomogeneity in sample thickness (different thicknesses are averaged to a single spectrum to represent a realistic measurement scenario). 
In order to incorporate a varying phase of the incident light and to investigate the impact of the variation of the other parameters, a model was derived from electromagnetic theory for a three layer ${ }_{85}$ system (air, sample, reflective substrate). In this work, variations of all three parameters are modeled, separately at first, and simultaneously afterwards, to demonstrate the combination effect. The sample thickness considerations are extended to $12.5 \mu \mathrm{m}$ in order to take into account the range most commonly used in tissue analysis. The goal is to investigate the influence of the three effects on EFSW in a hypothetical absorber.

\section{${ }_{90}$ 2. Experimental section}

All calculations and simulations were done in the MatLab 7.10 environment (The MathWorks, Natick, MA) using in-house written scripts. Theoretical absorbance spectra were calculated (as explained in Section 3.2) for a range of 11 angles of incidence (step of $\left.1^{\circ}\right), 18$ phases $\left(20^{\circ}\right.$ increment) and for 121 thicknesses (0.5-12.5 $\mu \mathrm{m}$ with a step of $0.1 \mu \mathrm{m})$, giving a set of almost 24,000 spectra for each of the $\mathrm{s}$ ${ }_{95}$ and p polarizations. The final absorbance spectra were calculated as a mean of the two polarizations. Therefore, the final dataset comprised almost 72,000 spectra. The complex refractive indices of gold and silver were acquired from a database compilation of Palik [25]. Models for both substrates were calculated, but only data for gold are presented in this paper (results for silver were almost identical).

\section{Results and Discussion}

\subsection{The absorbing system}

The model of Bassan et al.[24] simulated spectra of a hypothetical IR absorber with sample thickness varying between 0.5 and $4.5 \mu \mathrm{m}$. For the current study, the hypothetical absorber was simulated in the same manner, to enable a direct comparison of the results. The absorption spectrum is composed of equally spaced Lorentzian peaks of equal heights and widths (Figure 1). The corresponding real and 105 imaginary parts of the absorber were obtained via the Kramers-Kronig relation and are given in the Electronic Supplementary Information in Figures (ESI).

\subsection{Model}

The model is based on simple consideration of a linearly polarized monochromatic planar wave incident on a three layer system, depicted in Figure 2. Such an approach is complete for

many different cases and could be extended to a multilayer system (as for example in Bassan et al. [24]) according to the scheme described in Hecht et al.[26] The plane of incidence is perpendicular to the layer surfaces. The electrical vector of the wave is constant in time and could be perpendicular to the plane ( $s$ polarized light) or could lie in the plane ( $p$ polarized light).

115

By applying boundary conditions, and for simplicity considering only the reflections indicated in Figure 2, it is possible to find the amplitude reflection coefficients for both polarizations $r_{s}$ and $r_{p}$ respectively:

$$
r_{s}=1-\frac{2 n_{2}\left(n_{2} \sin (\phi+\Delta) \cos \theta_{2}-i n_{3} \cos (\phi+\Delta) \cos \theta_{3}\right)}{n_{1} \cos \theta_{1}\left(n_{3} \sin (\phi+\Delta) \cos \theta_{3} / \cos \theta_{2}-i n_{2} \cos (\phi+\Delta)\right)-i n_{2}\left(n_{3} \cos (\phi+\Delta) \cos \theta_{3}+i n_{2} \sin (\phi+\Delta) \cos \theta_{2}\right)},
$$




$$
r_{p}=1+\frac{2 n_{1}\left(i n_{2} \cos (\phi+\Delta) \cos \theta_{3}-n_{3} \sin (\phi+\Delta) \cos \theta_{2}\right)}{n_{1}\left(n_{3} \sin (\phi+\Delta) \cos \theta_{2}-i n_{2} \cos (\phi+\Delta) \cos \theta_{3}\right)+n_{2} \cos \theta_{1}\left(n_{2} \sin (\phi+\Delta) \cos \theta_{3} / \cos \theta_{2}-i n_{3} \cos (\phi+\Delta)\right)}
$$

The notation in equations 1 and 2 is consistent with Figure 2 and $n_{1}, n_{2}, n_{3}$ are the refractive indices of air, the sample and the mirror. It is important to note that $n_{2}$ and $n_{3}$ are complex.

${ }_{125}$ The (variable) phase of the incident wave is $\Delta$ and the phase change $\phi$ is associated with the transmission of the wave through a sample with thickness $d$ :

$$
\phi=k_{0} d n_{2} \cos \theta_{2}
$$

where $k_{0}$ is a wave vector in vacuum.

${ }_{130}$ In order to obtain a final absorbance spectrum, a series of steps need to be taken. First, the amplitude reflection coefficients need to be transformed to reflectivity:

$$
R_{s}=r_{s} r_{s}^{*}
$$

The reflectivity of non-polarized light is taken as an average of $s$ and $p$ polarization reflectivities. Afterwards, a ratio of the system without the sample $\left(R_{\text {background }}\right)$ and with the sample $\left(R_{\text {sample }}\right)$ is ${ }_{135}$ calculated and transformed to absorbance $(A)$ (Equation 5):

$$
A=-\log _{10}\left(\frac{R_{\text {sample }}}{R_{\text {background }}}\right)
$$

\subsection{Dependence on overall thickness}

In order to validate the comparison of the model described above to the one used in the previous ${ }_{140}$ Work[24], predicted spectra for varying thickness are presented in Figure 3. In comparison with the study of Bassan et al.[24], the thickness range has been extended to take into account typical tissue section thicknesses $(0-20 \mu \mathrm{m})$.

Clearly, a pattern similar to the one obtained by Bassan et al. is evident in the simulated spectra. A periodic variation of both the background and peak intensities is seen across different samples. The ${ }_{145}$ variations in the background are expected to diminish with increasing sample thickness, as more nodes of the infrared radiation penetrate the sample. As seen in Figure 3, the relative variations in the background are diminished significantly, at 20,12.5 and even $9 \mu \mathrm{m}$. However, a dramatic variation of the absorption peaks is still apparent. A simulation of much higher thicknesses up to $500 \mu \mathrm{m}$ was performed (data not shown) to determine at what range the EFSW becomes negligible in terms of this ${ }_{150}$ peak variation, indicating that a thickness of the order of 50-100 micrometers is required. This scenario is not relevant to infrared spectroscopy of biological samples, since at 15-20 $\mu \mathrm{m}$ little or no light is transmitted by the sample in transflection mode (for typical biological samples).

\subsection{Averaging over angle of incidence}


Variation of the angle of incidence changes the effective optical path length and therefore the EFSW. Experimentally, the source is delivered to the sample by the Cassegrain objective over a range of $\sim 35^{\circ}$ $-45^{\circ}$, and thus the EFSW should be integrated over the full angular range. Figure 4 shows the simulated spectra for 3 out of 11 different angles of incidence over the range, $35^{\circ}-45^{\circ}$ for a selected thickness of 1604.5 micrometers. Adopting the approach of Bassan et al., a single average value of the refractive index was assumed for all angles[24]. As expected, different angles give rise to different EFSW patterns and, as a result, the mean spectrum over all angles (Figure 4, blue) has smaller amplitude of peak maxima changes than the spectrum at the mean angle $40^{\circ}$. It should be noted, however, that the effects of a uniform intensity over all angles has been modeled, whereas experimentally the intensity profile will ${ }_{165}$ vary with angle. Nevertheless, this averaging does not significantly diminish the impact of the EFSW on the relative heights of the absorption maxima.

\subsection{Averaging over phase}

It should be noted that previous models of the EFSW considered effectively the case of a single, fully coherent wave, while in reality sources of infrared radiation are at best quasi-coherent (synchrotron or ${ }_{170}$ Globar). Moreover, for a given wavelength, one should take into consideration all possible node positions and not only the case of a node directly on the reflective substrate surface. As a result, the EFSW should be further averaged out. The analysis of Figure 5 demonstrates that, for multiple individual waves of varying incident phase, the combined EFSW effect is reduced compared to that of multiple coherent waves of the same phase. The effect of this kind of averaging is shown in Figure 5. ${ }_{175}$ Similar to the case of angle of incidence, an averaging effect is apparent. The overall amplitude changes are smaller, although, for the peak close to $1000 \mathrm{~cm}^{-1}$, an increase is seen. Nevertheless, the effect of considering a distribution of incident wave phases is a reduction of effect on the EFSW.

\subsection{Thickness averaging}

When trying to arrive at a more realistic representation of the EFSW, one needs to consider that every 180 sample intrinsically has an inhomogeneity in sample thickness. As a consequence, either in single point or in imaging mode, an averaging effect occurs, whenever the thickness variations over the sampled are significant (which is the case in FT-IR of tissues). Moreover, in preparing tissue sections for measurements by using a microtome, it is impossible to cut a section at exactly e.g. $4.5 \mu \mathrm{m}$. The manufacturers provide a range of error of $1 \mu \mathrm{m}$ in a 2-20 $\mu \mathrm{m}$ cutting range [27]. Furthermore, parts of

185 the tissue will inherently have different elasticity and will be cut in different ways. This fact has even further implications, since a drying section will change its thickness according to its elasticity parameters, as has been recently shown by AFM measurements [28]. The effects on the EFSW of a 1 $\mu \mathrm{m}$ variation on a spectrum of a $1.5 \mu \mathrm{m}$ sample and 2.5 variation on $10 \mu \mathrm{m}$ sample are shown in Figure 6.

190

The net effect is seen in the mean spectra of the respective thicknesses. The background oscillation is almost completely removed and what is even more important, so is the amplitude variations of peak maxima in the sample of 10 micrometers. The original hypothetical absorber profile is almost perfectly retrieved despite the EFSW in the system. This result has profound implications. Whenever any 
${ }_{195}$ Surface roughness with a lateral spatial frequency of order of the spot size is present, the resultant EFSW will be strongly diminished. It is especially clear with higher sample thickness. It is therefore of interest to consider whether such strong averaging is present in lower thickness samples. The 0.5-2.5 micrometers range shows that it is not as perfect. The reason for this is seen in the $3 \mathrm{D}$ plots (additional data plots for insight given in ESI: Figures $3 \mathrm{~S}-6 \mathrm{~S}$ ) - for larger thicknesses, the peak maxima 200 oscillations are changing rapidly, while it is not the case in the lower range. In the end, the effect will strongly depend on whether a sufficient number of oscillations will be averaged out. Results for a set of different ranges are shown in Figure 7 along with spectra for all calculated thicknesses.

It can be seen that, for the range of 1.5-3.5 $\mu \mathrm{m}$, the averaging is still quite efficient, although not to the same extent for the thinner samples. The mean spectrum of the 0.5-1.5 $\mu \mathrm{m}$ sample still shows ${ }_{205}$ considerable effects of the EFSW. The reason for this lies in the fact that the background oscillation (and corresponding amplitude change oscillation) has too low frequency to effectively cancel out. This in turn, is a direct consequence of the number of nodes penetrating the sample. In order to check how large the inhomogeneities in sample thickness need to be, a $10 \%$ value was simulated for $10 \mu \mathrm{m}$ thickness. The resultant mean spectrum still shows strong averaging of EFSW, although some ${ }_{210}$ amplitude differences between peak maxima can be seen.

\subsection{A combination effect}

All three effects discussed above have been evaluated independent of each other and a combination of effects should be taken into account. The calculations were therefore repeated in such a way that, for 215 each thickness, all theta angles and all phases were also calculated. A mean along all three parameters can be considered as a combination effect and is shown for a 7.5-12.5 $\mu \mathrm{m}$ sample in Figure 8 .

The incorporation of all three parameters at once even further improves the simulated spectrum of a hypothetical absorber. The combination effect was also calculated for other thickness ranges of interest and is shown in Figure 9.

${ }_{220}$ Again the mean spectra of samples in the range $>2 \mu \mathrm{m}$ are very close to the original hypothetical absorber. The spectral range most variable is the one associated with longest wavelength, i.e. close to $1000 \mathrm{~cm}^{-1}$, due to inherently fewer nodes at this wavelength penetrating the sample.

\subsection{Final discussion}

225

Previous studies of the detrimental impact of the EFSW on FTIR spectra of biological samples in transflection geometry have raised alarm bells about the use of the technique and critically the validity of the result of previous studies, present in the literature of the past 20 years. Indeed, the potential concerns were highlighted in the Editorial of a recent themed journal edition [29]. Diem et al. [30] have argued ${ }_{230}$ that appropriate data pre-processing can alleviate the confounding factors of the EFSW. Furthermore, a very recent work by Cao et al. [31] showed that for cell cultures prepared on transmission and on 
transflection substrates, no statistically significant discrimination due to EFSW was seen, indicating that the intrinsic cellular variations were more significant than any experimental variations due to EFSW.

In the study presented here, the model of Bassan et al. has been extended to encompass variations in ${ }_{235}$ three experimental parameters, in an effort to improve the validity of the simulated effects of the EFSW on spectra. It is clear that, for each variable, an averaging effect results, which can at least in part account for the discrepancies between the simulated and experimentally observed EFSW of Bassan et al.[24] Out of the three parameters considered, averaging of thickness inhomogeneities was found to have the most prominent impact on the effects of EFSW on the modeled absorption spectrum. The averaging renders ${ }_{240}$ the EFSW negligible for samples with higher thickness. It is important to note, however, that the low thickness regime ( $<2$ micrometers) is less influenced by the averaging effects. Critical, however, is the thickness variation in comparison to the spatial resolution of the imaging system. The latter is obtained from a single pixel of focal plane detector of $5.5 \mu \mathrm{m} \times 5.5 \mu \mathrm{m}$ and is of the order of the wavelength, approximately 5-10 $\mu \mathrm{m}$ in the fingerprint region of the spectrum. Notably, variations of tissue section ${ }_{245}$ surface structures have been demonstrated to occur precisely within this length scale [28, 32].

Another important point is raised by the simulation. It is that the oscillation of peak maxima amplitude is inversely proportional to frequency for a fixed sample thickness, and therefore, if there is a large change in amplitude as a result of EFSW, then it will be most prominent when comparing peaks of large spectral differences (e.g. $\mathrm{CH}_{2} / \mathrm{CH}_{3}$ stretching at 2800-3000 $\mathrm{cm}^{-1}$ and amide $\mathrm{I}$ band at $1650 \mathrm{~cm}^{-1}$ ), but at short ${ }_{250}$ spectral differences the relative amplitude does not change as dramatically. This means that when comparing amide I and II bands or their shapes, the expected changes in relative amplitudes are quite small. The latter conclusion has other implications - the small changes in relative peak amplitudes may impact on apparent peak positions (on the order of the spectral resolution) but will not result in significant shifts of for example $10 \mathrm{~cm}^{-1}$. This kind of spectral behavior should not be attributed to ${ }_{255}$ EFSW.

\section{Conclusions}

Despite the simplicity of the model presented here, it is possible to see that many parameters should be considered for understanding the spectral data from an FTIR transflection experiment. Out of three potential averaging effects identified here, the thickness averaging can play a pivotal role in significantly ${ }_{260}$ lowering the EFSW in transflection FT-IR spectra, when the lateral length scale of the spatial variations are of similar dimensions to the spatial resolution of the measurement. This is a case for imaging of many tissue cross-sections, but maybe not relevant when in vitro single cells are measured. Angle and phase averaging also have a significant effect on the EFSW and along with thickness averaging for samples above $2 \mu \mathrm{m}$ of thickness, the EFSW is almost completely removed.

${ }_{265}$ The results presented here may have significant impact on the future of biomedical applications of FTIR spectroscopy. Consideration of the averaging effects for sample inhomogeneities, source incoherence, and range of angles of incidence indicate that, in samples of sufficient thickness $(\sim 5 \mu \mathrm{m})$, the distorting effects of EFSW become negligible, implying that the use of low cost low e substrates may yet be acceptable. Furthermore and critically, the results indicate that the significant body of work over the past ${ }_{270}$ two decades on such substrates is not necessarily invalid.

\section{Acknowledgements}


This work was supported by the European Union under the European Regional Development Fund (grant coordinated by JCET-UJ, POIG.01.01.02-00-069/09).

\section{${ }_{280}$ References}

[1] B. Mohlenhoff, M. Romeo, M. Diem, B.R. Wood, Biophysical journal, 88 (2005) 3635-40.

[2] B. Bird, M. Miljković, M. Diem, Journal of biophotonics, 3 (2010) 597-608.

[3] M. Miljković, B. Bird, M. Diem, The Analyst, 137 (2012) 3954-64.

[4] K.R. Bambery, B.R. Wood, D. McNaughton, The Analyst, 137 (2012) 126-32.

${ }_{285}$ [5] P. Bassan, A. Sachdeva, A. Kohler, C. Hughes, A. Henderson, J. Boyle, J.H. Shanks, M. Brown, N.W. Clarke, P. Gardner, The Analyst, 137 (2012) 1370-7.

[6] P. Bassan, A. Kohler, H. Martens, J. Lee, H.J. Byrne, P. Dumas, E. Gazi, M. Brown, N. Clarke, P. Gardner, The Analyst, 135 (2010) 268-77.

[7] P. Bassan, A. Kohler, H. Martens, J. Lee, E. Jackson, N. Lockyer, P. Dumas, M. Brown, N. Clarke, P. ${ }_{200}$ Gardner, Journal of biophotonics, 3 (2010) 609-20.

[8] B.R. Wood, T. Chernenko, C. Matthaus, M. Diem, C. Chong, U. Bernhard, C. Jene, A.A. Brandli, D. McNaughton, M.J. Tobin, A. Trounson, O. Lacham-Kaplan, 80 (2008) 9065-9072.

[9] P. Lasch, W. Haensch, D. Naumann, M. Diem, Biochimica et biophysica acta, 1688 (2004) 176-86.

[10] E. Ó Faoláin, M.B. Hunter, J.M. Byrne, P. Kelehan, M. McNamara, H.J. Byrne, F.M. Lyng, ${ }_{295}$ Vibrational Spectroscopy, 38 (2005) 121-127.

[11] K.L.A. Chan, S.G. Kazarian, Analytical chemistry, 85 (2013) 1029-36.

[12] R. Bhargava, Analytical and bioanalytical chemistry, 389 (2007) 1155-69.

[13] R. Bhargava, D.C. Fernandez, S.M. Hewitt, I.W. Levin, Biochimica et biophysica acta, 1758 (2006) $830-45$.

${ }_{300}$ [14] A. Lattermann, C. Matthäus, N. Bergner, C. Beleites, B.F. Romeike, C. Krafft, B.R. Brehm, J. Popp, Journal of biophotonics, 12 (2012) 1-12.

[15] P. Lasch, M. Boese, A. Pacifico, M. Diem, Vibrational Spectroscopy, 28 (2002) 147-157. 
[16] A.D. Meade, F.M. Lyng, P. Knief, H.J. Byrne, Analytical and bioanalytical chemistry, 387 (2007) $1717-28$.

${ }_{305}$ [17] T.P. Wrobel, K. Majzner, M. Baranska, Spectrochimica Acta Part A: Molecular and Biomolecular Spectroscopy, (2012).

[18] T.P. Wrobel, K.M. Marzec, K. Majzner, K. Kochan, M. Bartus, S. Chlopicki, M. Baranska, The Analyst, 137 (2012) 4135-9.

[19] B.R. Wood, K.R. Bambery, C.J. Evans, M. a Quinn, D. McNaughton, BMC medical imaging, 6 ${ }_{310}(2006) 12$.

[20] T.P. Wrobel, L. Mateuszuk, R.B. Kostogrys, S. Chlopicki, M. Baranska, Analyst, DOI: 10.1039/C3AN01050C (2013).

[21] H. Brooke, B. V Bronk, J.N. McCutcheon, S.L. Morgan, M.L. Myrick, Applied spectroscopy, 63 (2009) 1293-302.

${ }_{315}$ [22] J. Filik, M.D. Frogley, J.K. Pijanka, K. Wehbe, G. Cinque, The Analyst, 137 (2012) 853-61.

[23] K. Wehbe, J. Filik, M.D. Frogley, G. Cinque, Analytical and bioanalytical chemistry, 405 (2013) $1311-24$.

[24] P. Bassan, J. Lee, A. Sachdeva, J. Pissardini, K.M. Dorling, J.S. Fletcher, A. Henderson, P. Gardner, The Analyst, 138 (2012) 144-157.

${ }_{320}$ [25] M. Roman, A. Kaczor, M. Baranska, Journal of molecular modeling, 16 (2010) 1549-57.

[26] A. Jaworska, R. Wietecha-Posłuszny, M. Woźniakiewicz, P. Kościelniak, K. Malek, The Analyst, 136 (2011) 4704-9.

[27] M. Zimmermann, S. Lange, J. Lampe, I. Smirnow, M. Bitzer, U.M. Lauer, http://www.leicamicrosystems.com/uploads/media/white_paper_vt1200s_slicing_of_liver_tissue_email.pdf.

${ }_{325}$ [28] K.M. Marzec, T.P. Wrobel, A. Rygula, E. Maslak, A. Jasztal, A. Fedorowicz, S. Chlopicki, M. Baranska, in review, 0-33.

[29] P. Heraud, B.R. Wood, The Analyst, 138 (2013) 3861-2.

[30] M. Miljković, B. Bird, K. Lenau, A.I. Mazur, M. Diem, The Analyst, (2013) 3975-3982.

[31] J. Cao, E.S. Ng, D. McNaughton, E.G. Stanley, A.G. Elefanty, M.J. Tobin, P. Heraud, The Analyst, ${ }_{330}(2013)$ 4147-4160.

[32] D. a Lamprou, V. Venkatpurwar, M.N.V.R. Kumar, PloS One, DOI:10.1371/journal.pone.0064490 (2013). 


\section{Figure Captions}

340

Figure 1. A hypothetical absorber - peaks of equal heights and widths.

Figure 2. Model three layer system. The electric $(\vec{E})$ and propagation vectors $(\vec{k})$ are depicted. Indices $p$ and $s$ describe perpendicular and parallel polarizations, $i$ and $r$ incident and refracted wave, numbers 1,2 , ${ }_{345} 3$ represent propagation in the first, second and third layers with refractive indices $n_{1}, n_{2}$ and $n_{3}$.

Figure 3. Simulation of absorbance spectra of a hypothetical absorber with different sample thickness EFSW is evident in all cases.

${ }_{350}$ Figure 4. Simulated spectra of three out of 11 different incidence angles $\left(35^{\circ}-45^{\circ}\right)$ and their mean (blue).

Figure 5. Simulated spectra of three phases out of 18 different phases $\Delta$ from 0 to $2 \pi$ and their mean (blue).

Figure 6. 3D plots (left) of all spectra within the averaged range and the corresponding effects of thickness averaging (right) on EFSW in samples of 10 (7.5-12.5) and 1.5 (0.5-2.5) micrometer thickness. 355

Figure 7. Thickness averaging and the effects of different ranges and different surface roughness on the EFSW present in the simulated samples.

Figure 8. A combination effect taking into account all three parameters (incidence angle, phase and thickness) at once compared with a spectrum averaged over thickness only.

${ }_{360}$ Figure 9. A combination effect of EFSW averaging for different thicknesses ranges. 